\title{
3D TOUCHLESS FINGERPRINTS: COMPATIBILITY WITH LEGACY ROLLED IMAGES
}

\author{
Yi Chen ${ }^{1}$, Geppy Parziale ${ }^{2}$,Eva Diaz-Santana ${ }^{2}$, and Anil K. Jain ${ }^{1}$ \\ ${ }^{1}$ Michigan State University \\ Department of Computer Science and Engineering \\ East Lansing, Michigan, 48824 \\ ${ }^{2}$ TBS Holding AG \\ Schindellegistrasse 19 \\ CH-8808, Pfaeffikon, Switzerland
}

\begin{abstract}
Fingerprints are traditionally captured based on contact of the finger on paper or a platen surface. This often results in partial or degraded images due to improper finger placement, skin deformation, slippage and smearing, or sensor noise from wear and tear of surface coatings. A new generation of touchless live scan devices that generate $3 \mathrm{D}$ representation of fingerprints is appearing in the market. This new sensing technology addresses many of the problems stated above. However, $3 \mathrm{D}$ touchless fingerprint images need to be compatible with the legacy rolled images used in Automated Fingerprint Identification Systems (AFIS). In order to solve this interoperability issue, we propose a unwrapping algorithm that unfolds the $3 \mathrm{D}$ fingerprint in such a way that it resembles the effect of virtually rolling the $3 \mathrm{D}$ finger on a $2 \mathrm{D}$ plane. Our preliminary experiments show promising results in obtaining touchless fingerprint images that are of high quality and at the same time compatible with legacy rolled fingerprint images.
\end{abstract}

\section{INTRODUCTION}

An automated fingerprint authentication system consists of three components, namely, image acquisition, feature extraction and matching. Among the three, image acquisition is often considered the most critical as it determines the fingerprint image quality, which has a large effect on the system performance [1]. Traditionally, fingerprint images are acquired by pressing or rolling a finger against a hard surface (e.g., glass, silicon, polymer) or paper (e.g., index card). This often results in partial or degraded images due to improper finger placement, skin deformation, slippage and smearing, or sensor noise from wear and tear of surface coatings.

A number of companies are developing touchless sensing technology that performs "finger imaging" as opposed to conventional "finger printing" $[2,3]$. That is, the sensor images a finger from different views using a multi-camera system and reconstructs a contact-free 3D representation of the fingerprint. Touchless sensing technology provides an idea solution to the intrinsic problems of the contact-based technology as stated above and results in repeatable and high quality image acquisition. In addition, the reconstructed 3D fingerprint gives a much larger, nail-to-nail representation of the fingerprint, compared to conventional contact-based fingerprints.

3D touchless fingerprints, however, need to be compatible with the conventional contact-based 2D rolled fingerprint images used in Automated Fingerprint Identification Systems (AFIS). In order to make 3D touchless fingerprints interoperable with current AFIS systems, we introduce a simulated rolling procedure which essentially unwraps the $3 \mathrm{D}$ touchless fingerprints into 2D such that the resulting 2D fingerprints are comparable with legacy rolled fingerprints. This is a very challenging task because the simulated rolling procedure must not introduce distortions other than those compatible with the physical deformation of skin due to rolling. As a result, we propose "Equidistance Unwrapping" to minimize the distortion during unwrapping while preserving the "ground-truth" of the fingerprint.

The rest of the paper is organized as follows: Section $2 \mathrm{de}-$ scribes the procedure for $3 \mathrm{D}$ reconstruction of touchless fingerprints. Section 3 describes various unwrapping methods to unfold the 3D fingerprint to 2D, specifically, the cylindricalbased parametric unwrapping and the proposed non-parametric unwrapping method. In Section 4, we compare the two unwrapping methods by showing the final unwrapped images. Compatibility of the unwrapped and conventional rolled images is also shown on a small database. Conclusions and future work follow in Section 5.

\section{3D RECONSTRUCTION OF TOUCHLESS FINGERPRINTS}

Touchless fingerprinting is essentially a remote sensing technique used to capture the ridge-valley pattern. While it is not 
a completely new approach to acquire fingerprints [2, 3, 4], it did not generate a sufficient interest in the market, in spite of its advantages with respect to the contact-based technology. The main reason is the cost of this technology. In fact, in order to keep the production costs of these devices low, their manufacturers often use only one camera. This results in fingerprint images with less usable area, due to the curvature of the finger, compared to the contact-based approach. In a touchless fingerprint image, the apparent frequency of the ridge-valley pattern increases from the center towards the side until ridges and valleys become undistinguishable. Hence, dedicated algorithms are needed to correct the ridge-valley pattern with an increase in the overall computational load.

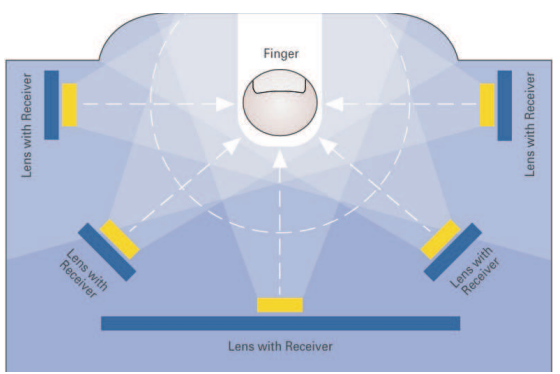

Fig. 1. Fingerprint acquisition using a set of cameras surrounding the finger.

The approach proposed in this paper is based on a multiview system. The use of multiple views enables the capture of the rolled-equivalent (full nail-to-nail) fingerprints that is faster than traditional rolling procedures [5], thereby increasing the usable fingerprint area. The different views can obtained by either different cameras surrounding the finger (Fig. 1) or one camera and a set of small mirrors (Fig. 2).

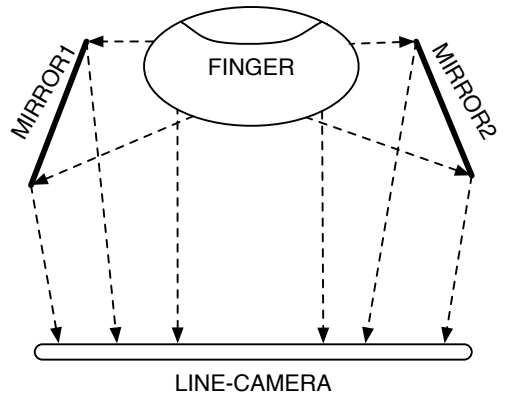

Fig. 2. Fingerprint acquisition obtained by combining a single line-scan camera and two side mirrors.

The exact position and orientation of each camera and mirror within a chosen reference coordinate system is computed using calibration algorithms [6, 7]. This information combined with the images of each view is used to generate a 3D finger model. For example, in the approach represented in Fig. 1, there are 5 cameras placed around the finger. From each acquired image, a silhouette is extracted. Knowing the position and orientation of each camera within a reference coordinate system, the 5 silhouettes are then projected into the 3D space and interpolated together obtaining the 3D shape of the finger (shape-from-silhouette). The 3D model is used to correct the perspective of the 5 original images, obtaining the corresponding ortho-images. Using the correlation between adjacent views, the five ortho-images are mosaicked, generating the first approximation of a rolled-equivalent fingerprint. As explained in the rest of this paper, the 3D model is then used to correct the geometry of the texture to preserve the distances between minutiae with respect to a rolled-equivalent fingerprint obtained by legacy devices.

\section{3D FINGERPRINT UNWRAPPING}

Generally, unwrapping a 3D object refers to the unfolding the 3D object onto a flat 2D plane. One important application of this problem - globe unwrapping (or map projection) has been heavily studied in Geographic Information Systems (GIS), since many technologies for working with geographic data are inherently flat, including paper and films [8,9]. Other applications of 3D object unwrapping include medical imaging, surface recognition and industrial design [10, 11, 12].

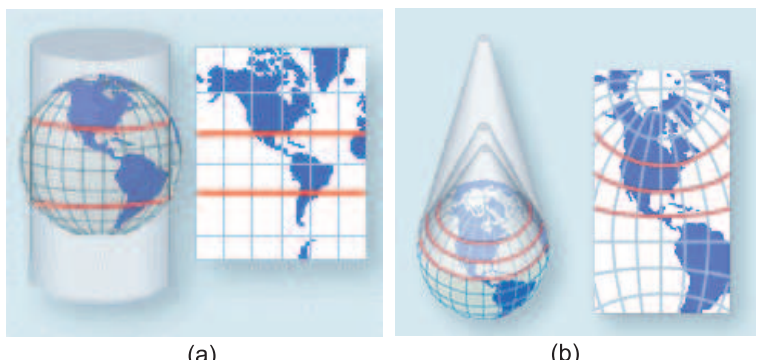

Fig. 3. Globe Unwrapping using (a) Cylindrical Model (b) Conic Model. Adopted from [13].

There are mainly two types of unwrapping methods, namely, parametric and non-parametric.

1. Parametric unwrapping refers to the projection of the 3D object onto a parametric model (i.e., cylindrical or conic) and the unwrapping of the model. This method often involves simple and straightforward transformations. But it also requires that the chosen parametric model be close to the shape of the object. Otherwise, large distortions may be introduced during unwrapping.

2. Non-parametric unwrapping, on the other hand, does not involve any projection on parametric models. Instead, the unwrapping directly applies to the object to preserve local distances or angular relations. This method is often employed for irregular-shaped objects. 
Figure 3 shows the unwrapping of the globe using two different parametric models: cylindrical and conic. It has to be noted that it is impossible to unwrap the $3 \mathrm{D}$ sphere to a 2D plane without introducing some distortion. One can only try to minimize the distortion by using multiple models for different portions of the object to best approximate the shape locally, as shown in the figure. In the case of 3D fingerprint unwrapping, this limitation also applies because although human fingers can be approximated as a cylinder or cone, distortion is still unavoidable, especially at the fingertip. In the rest of this section, we will give two examples of 3D fingerprint unwrapping, including a parametric method using cylindrical model and a nonparametric method based on equidistance. We will compare the two methods and show that distortion introduced by the parametric method can be noticeably large, whereas the nonparametric method demonstrates more faithful representation of the "ground-truth" of the fingerprint.

\subsection{Parametric 3D Fingerprint Unwrapping}

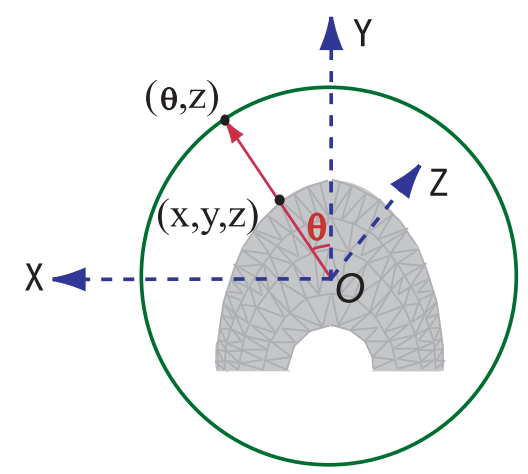

Fig. 4. Parametric unwrapping using a cylindrical model (topdown view). Point $(\mathrm{x}, \mathrm{y}, \mathrm{z})$ on the $3 \mathrm{D}$ finger is projected to $(\theta$, $\mathrm{z})$ on the 2D plane.

Human fingers vary in shape, for example, the shape of the middle finger is often more cylindrical than the thumb. Nevertheless, it is generally true that human fingers can be closely approximated by cylinders and hence, a cylindrical model is a reasonable choice for parametric unwrapping of 3D fingerprints. Human fingers also vary in size and the cylindrical model can also capture this size variability in the vertical direction, but not in the horizontal direction.

A simple illustration of the cylindrical-based unwrapping is to imagine projecting the fingerprint texture onto a cylinder surrounding the finger, and then unwrapping (flattening) the cylinder to obtain the $2 \mathrm{D}$ fingerprint. Mathematically, let the origin be positioned at the bottom of the finger, centered at the principle axis of the finger. Let $T$ be a point on the surface of the 3D finger:

$$
T=\left[\begin{array}{l}
x \\
y \\
z
\end{array}\right]
$$

This 3D point is then projected (transformed) onto the cylindrical surface to obtain the corresponding 2D coordinates $S$

$$
S=\left[\begin{array}{l}
\theta \\
z
\end{array}\right]
$$

where

$$
\theta=\tan ^{-1}\left(\frac{x}{y}\right)
$$

A top-down view of the unwrapping model is shown in Figure 4, where the $Z$ axis points outward from the origin. It must be noted that the finger is represented as a triangular mesh after $3 \mathrm{D}$ reconstruction and each vertex on a triangle would be directly projected using the above transformation. As a result, each triangle on the $3 \mathrm{D}$ finger would be mapped to a triangle on the cylinder, whereas points in between vertices of the triangle would be mapped using a linear interpolation.

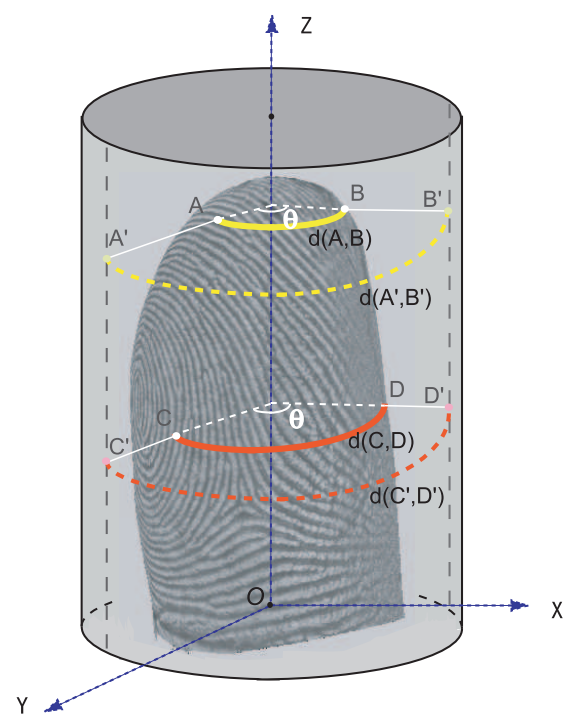

Fig. 5. Fingerprint unwrapping using the cylindrical model. Relative distances between points on the finger surface are not preserved after the unwrapping procedure.

Parametric fingerprint unwrapping using cylindrical model is efficient and straightforward, however, it does not preserve the relative distances between points on the finger surface. Figure 5 provides a visual illustration of the problem. For example, the surface distance $d(A, B)$ between points $A$ and $B$ at the fingertip is much smaller than that between points $C$ and $D(d(C, D))$ near the bottom, but since they both correspond to the same angle $\theta$, the unwrapped distances $d\left(A^{\prime}, B^{\prime}\right)$ and $d\left(C^{\prime}, D^{\prime}\right)$ become equal. In general, each cross section of the finger, big or small, is projected into a fixed-length row in the projected 2D image. As a result, horizontal distortion is introduced as the fingerprint will be noticeably stretched, especially at the fingertip, as shown in Figure 9(a).

In addition to the large stretching effects, parametric unwrapping often has limitations in preserving the size of the 
finger. Using the cylindrical model as an example, the mapping in the horizontal direction is based on the angle rather than the surface distance, and hence, size differences between fingers in the horizontal direction will be normalized out after the unwrapping. This results in another problem for preserving the "ground-truth" of fingerprints since horizontal scales in fingerprints are not retained after unwrapping.

\subsection{Non-parametric 3D Fingerprint Unwrapping}

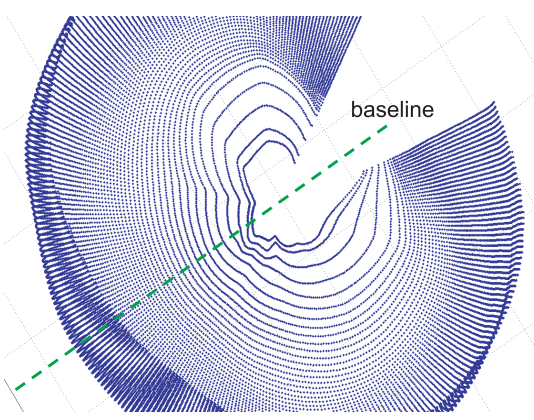

Fig. 6. 3D representation of the finger. Vertices of the triangular mesh are naturally divided into slices.

In the non-parametric approach, no parametric model is used for projection and the unwrapping is directly applied to an object with arbitrary shape such that some spatial properties in the object are preserved. As an example, one may want to preserve the geodesic distance between any two points in a local region on the object surface. This is a desirable property for our application on fingerprints because the matching of fingerprints is often performed based on the distances between minutiae (ridge bifurcation and ending) points. If we can preserve the distances between minutiae points in the unwrapped touchless fingerprints, the problem of interoperability between touchless and contact-based fingerprints is then reduced to skin deformation. Since no parametric model is used, this method also guarantees that the variability in both shape and size of fingers is preserved.

The essential idea of the non-parametric method we propose is to "locally unfold" the finger surface such that both inter-point surface distances and scale are preserved to a maximum degree. More specifically, for a given 3D finger, we divide it into thin parallel slices, orthogonal to the principle axis of the finger, and unfold each slice with a minimum amount of stretching. Because human fingers have very smooth structure, as long as each slice is sufficiently thin, the resulting unwrapped fingerprint texture will be smooth.

Figure 6 shows the triangular mesh representation of a 3D finger, where only vertices (no lines) of triangles are shown. Note that these vertices naturally form slices at different heights of the finger. However, distances between slices are too large to obtain a smooth unwrapping. As a result, linear interpolation is used to first extract more slices in between the vertices and create a more dense representation.

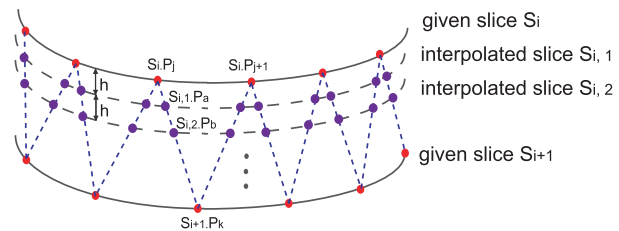

Fig. 7. Slice interpolation. We interpolate between given slices with a step-size $h$ to obtain finer representation in the vertical direction for unwrapping.

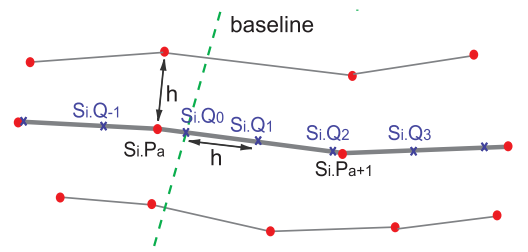

Fig. 8. Equidistant Resampling. We resample points on each slice with equal distance $h$ to obtain finer representation in the horizontal direction for unwrapping. The baseline defines the central column that the fingerprint will be mapped to.

Let $S_{i}$ and $S_{i+1}$ be the given slices from the triangular mesh and $h$ be the step-size (distance between slices in the dense representation) for interpolation. Figure 7 gives an illustration of the procedure. Let $S_{i} . P_{j}, S_{i} . P_{j+1}$ and $S_{i+1} . P_{k}$ be the three vertices of a given triangle. The position of the interpolated point $S_{i, 1} \cdot P_{a}$ is obtained as follows:

$$
\begin{aligned}
S_{i, 1} \cdot P_{a} \cdot x & =t \times S_{i+1} \cdot P_{k} \cdot x+(1-t) \times S_{i} \cdot P_{j} \cdot x \\
S_{i, 1} \cdot P_{a} \cdot y & =t \times S_{i+1} \cdot P_{k} \cdot y+(1-t) \times S_{i} \cdot P_{j} \cdot y \\
S_{i, 1} \cdot P_{a} \cdot z & =S_{i} \cdot P_{j} \cdot z+h,
\end{aligned}
$$

where

$$
t=\frac{S_{i, 1} \cdot P_{a} \cdot z-S_{i} \cdot P_{j} \cdot z}{S_{i} \cdot P_{j} \cdot z-S_{i+1} \cdot P_{k} \cdot z}
$$

is the proportion parameter. This procedure goes on for every step-size $h$ in height (z-axis); each slice in the final dense representation corresponds to a row in the final unwrapped fingerprint image.

Once a dense representation in height has been established, we apply similar interpolation on each slice to resample points at equal distance $h$ such that the neighboring points of the same slice would correspond to neighboring columns of the same row in the final unwrapped image. This step-size $h$ is equal to that in the vertical direction, and hence, the scale of the finger is properly preserved. A baseline, or starting point to unfold at each slice, is also defined as the intersecting line (curve) between the 3D finger and a plane passing through the principal axis at the center of the finger. That is, the direction of unwrapping is established from the center of the finger to the nail side. The resampling procedure is illustrated in Figure 8 and described by the following algorithm: 


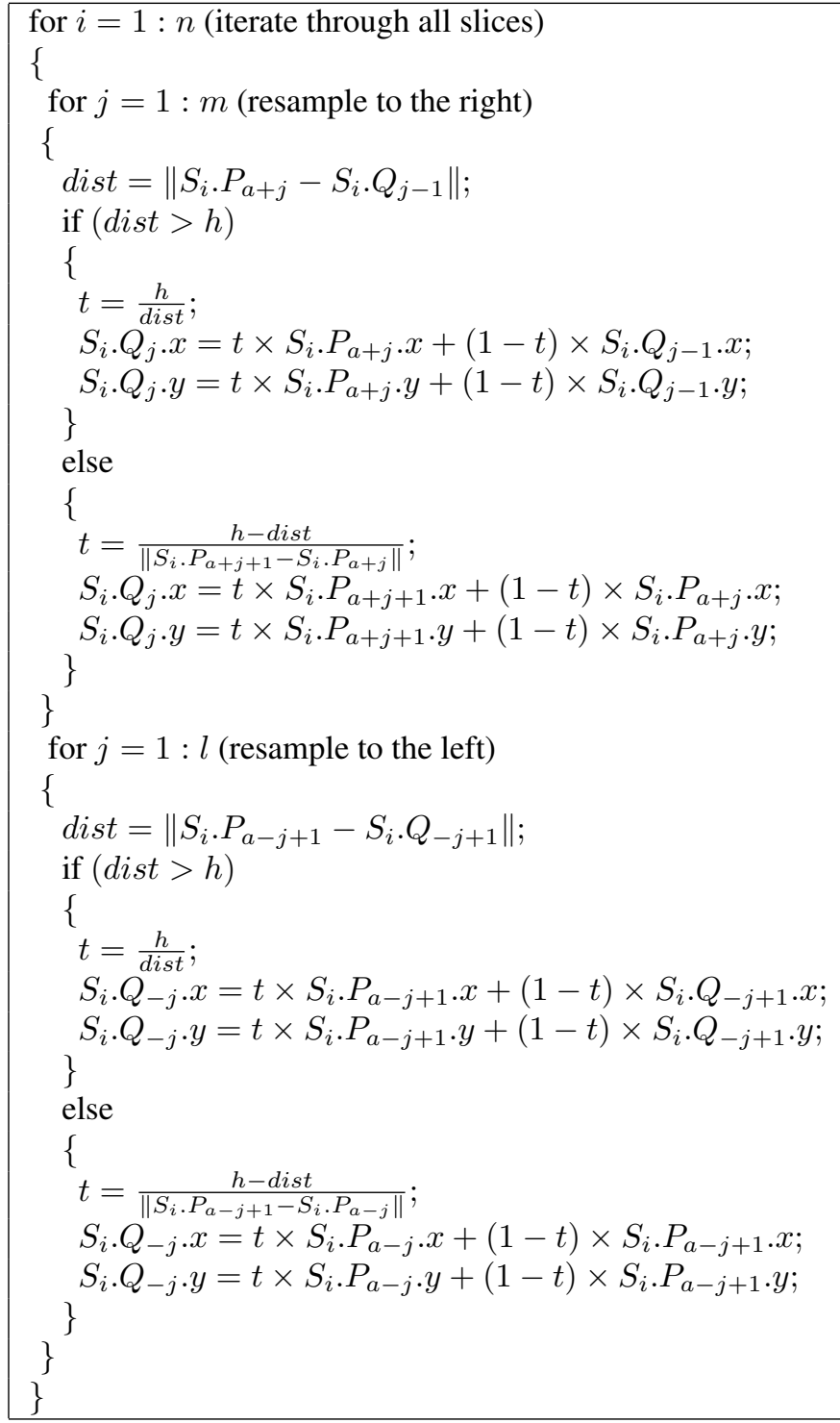

\section{EXPERIMENTAL RESULTS}

Figures 9(a) and (b) show the unwrapped touchless fingerprint images using the cylindrical-based and the proposed method, respectively. Minutiae points (white arrows) are extracted using the feature extraction algorithm in [14] and distances between a few minutiae points (red solid lines) are shown in Figures 9. These figures show that the proposed unwrapping method better preserves the inter-point distances with less distortion than the cylindrical-based method.

To demonstrate the compatibility of the unwrapped touchless fingerprints with legacy rolled images, we have collected a small database with 38 fingers, each includes one ink-onpaper rolled print and one touchless print using the new linescan sensor (at 1000 ppi) (see Figure 10).

A commercial fingerprint matching software was then used to evaluate the matching performance. Note that touchless fingerprints have lower contrast between ridges and valleys than legacy rolled images, hence, additional preprocessing of

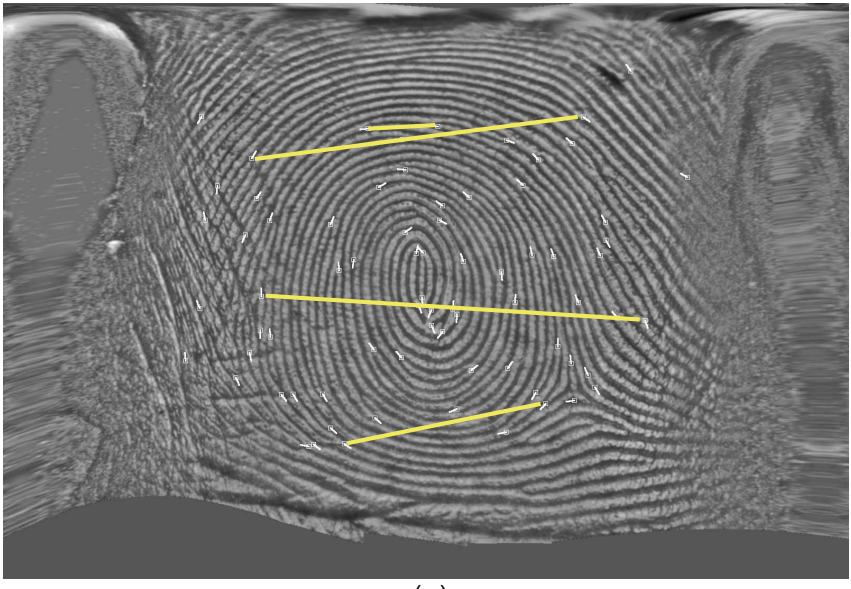

(a)

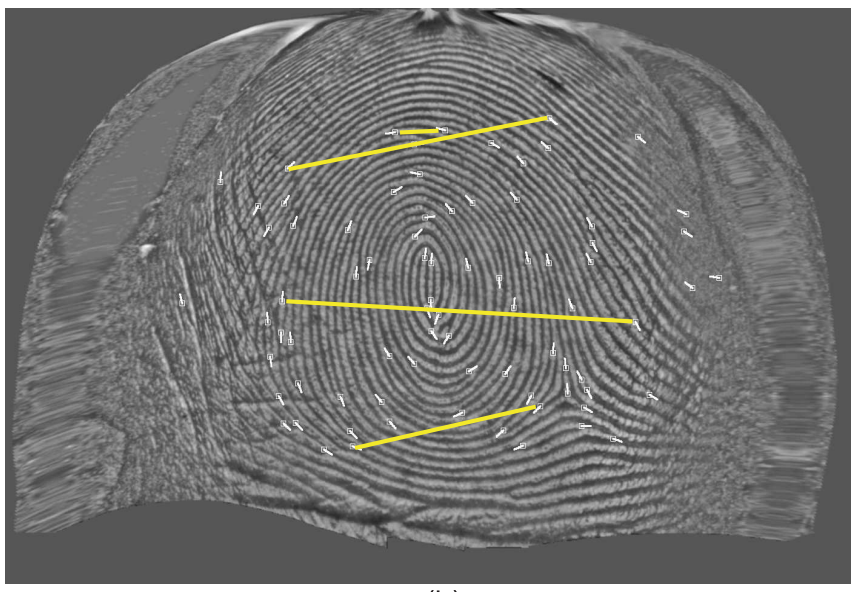

(b)

Fig. 9. Unwrapping a 3D fingerprint captured with Surround Imager using (a) the cylindrical-based parametric method and (b) the proposed non-parametric method. Note the stretching effects at the fingertip in (a) is minimized in (b). The 2D unwrapped fingerprint in (b) is more faithful to the "groundtruth" as it better retains the distances (red solid lines) between minutiae points (white arrows) than that in (a).

the touchless fingerprints was done before matching. Figure 11 shows the match score distributions after matching touchless fingerprint with ink-on-paper rolled fingerprint images. In total, there are 38 genuine scores and 2,812 impostor scores, which includes all between-group and withingroup impostor scores. There is almost no overlap (only one genuine score $(7,483)$ is below the maximum impostor score $(7,725))$ between genuine and impostor score distributions, suggesting compatibility between touchless and ink-on-paper rolled fingerprints in the given small database.

\section{CONCLUSIONS AND FUTURE WORK}

Although the unwrapped fingerprint images using our proposed method are faithful to the "ground-truth", they are not 


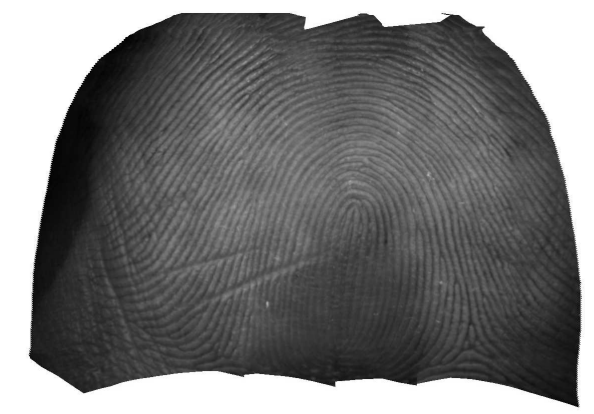

(a)

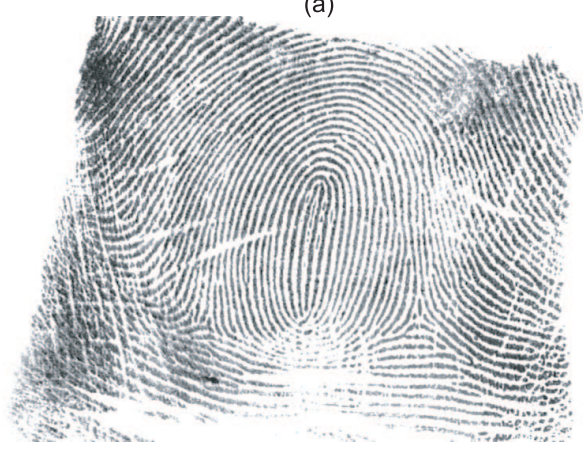

(b)

Fig. 10. Visualizing compatibility between (a) a touchless fingerprint from line-scan sensor using the proposed nonparametric unwrapping (b) the corresponding ink-on-paper rolled fingerprint.

yet completely compatible to the legacy rolled fingerprints. This is because the unwrapped fingerprints are touchless, or deformation free, whereas the legacy rolled fingerprints involve noticeable skin deformation caused by the rolling. Therefore, in our future work, we would like to explore the possibility of introducing skin deformation into our unwrapping model to generate unwrapped fingerprints that are truly rolledequivalent. A larger database is also being collected for a large-scale evaluation of compatibility between unwrapped touchless fingerprints and legacy rolled fingerprints.

\section{ACKNOWLEDGEMENTS}

The authors would like to thank Prof. Casim Abbas, Dirk Colbry, Unsang Park and Xiaoguang Lu for valuable discussions on 3D data analysis. This work is funded by NIJ grant 2005-IJ-CX-K701.

\section{REFERENCES}

[1] Tabassi E., Wilson C., and Watson C., "Fingerprint Image Quality," Tech. Rep. 7151, National Institute of Standards and Technology (NIST), August 2004.

[2] “TBS Touchless Fingerprint Sensor," http://www/tbsinc.com.

[3] "Mitsubishi Touchless Fingerprint Sensor," http://global.mitsubishielectric.com.

[4] “TST Group,” http://www.tst-ag.de.

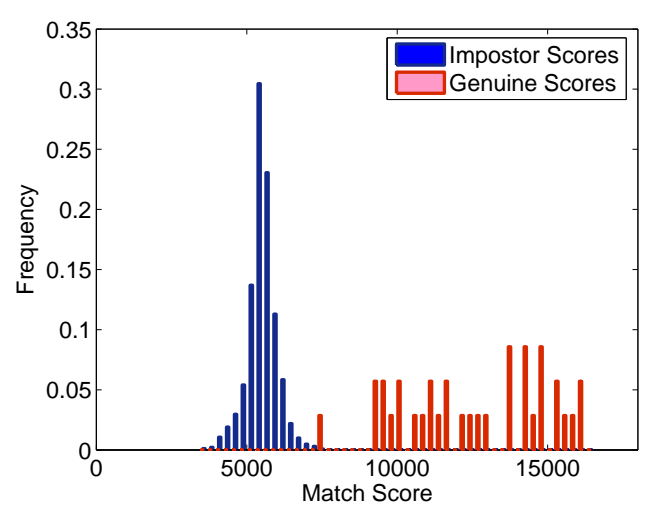

Fig. 11. Matching touchless with ink-on-paper fingerprints. Genuine and Impostor match scores are well separated with only one genuine score $(7,483)$ below the maximum impostor score $(7,725)$, indicating that touchless fingerprints are compatible with legacy rolled fingerprint images.

[5] Parziale G. and Diaz-Santana E., "The Surround Imager: a Multi-Camera Touchless Device to Acquire 3D Rolled-Equivalent Fingerprints," in Proceedings of IAPR International Conference on Biometrics (ICB), Hong Kong, China, January 2006, pp. 244-250.

[6] Tsai, R., "An Efficient and Accurate Camera Calibration Technique for 3D Machine Vision," in Proceedings of IEEE Conference on computer Vision and Pattern Recognition, Florida, USA, 1986, pp. 364-374.

[7] Z. Zhang, "Flexible camera calibration by viewing a plane from unknown orientations," IEEE Transactions on Pattern Analysis and Machine Intelligence, vol. 11, pp. 1330-1334, 2000.

[8] Snyder J. P., Flattening the Earth: Two Thousand Years of Map Projections, The University of Chicago Press, 1993.

[9] Yang O., Tobler W., Snyder J., Yang Q. H., Map Projection Transformation, Taylor \& Francis, 2000.

[10] Kita Y., Wilson D. L., and Noble J. A., "Real-time Registration of 3D cerebral Vessels to X-ray Angiograms," in Proceedings of International Conference on Medical Image Computing and Computer-Assisted Intervention, Cambridge, MA, Octobor 1998, pp. 1125-1133.

[11] Summers D., Texturing: Concepts and Techniques, Charles River Media Inc., 2004.

[12] Glassner A. S., Graphics Gems, Elsevier, 1998.

[13] "Map Projections: From Spherical Earth to Flat Map," http://nationalatlas.gov/articles/mapping/aprojections.html.

[14] Jain A. K., Hong L., and Bolle R., "On-line Fingerprint Verification," IEEE Transactions on Pattern Analysis and Machine Intelligence, vol. 19, no. 4, pp. 302-314, April 1997. 